\title{
An important functional group, benzo[1,3]dioxole, of alkaloids induces the formation of the human telomeric DNA G-quadruplex
}

\author{
YANG Shu $^{1,2}$, XIANG JunFeng ${ }^{1 *}$, YANG QianFan ${ }^{1,2}$, ZHOU QiuJu $^{1,2}$, ZHANG XiuFeng $^{1}$, \\ Li Qian ${ }^{1,2}$, TANG YaLin $^{1 *} \&$ XU GuangZhi ${ }^{1}$ \\ ${ }^{1}$ Beijing National Laboratory for Molecular Sciences, Centre for Molecular Sciences, State Key Laboratory for Structural Chemistry for Unstable \\ and Stable Species; Institute of Chemistry, Chinese Academy of Sciences, Beijing 100190, China; \\ ${ }^{2}$ Graduate University of Chinese Academy of Sciences, Beijing 100049, China
}

Received February 4, 2010; accepted June 18, 2010

Over the past few decades, numerous molecules have been discovered or designed to interact efficiently and selectively with a peculiar DNA structure named G-quadruplex. Some of these molecules have been developed as anticancer agents. To aid the design of anticancer agents, the ability of alkaloids possessing Protoberberine and Benzophenanthridine groups to induce the formation of G-quadruplexes were studied using CD spectroscopy. By careful examination of their structures, we found that a benzo[1,3]dioxole group plays an important role in influencing their inductive properties. The more functional groups the alkaloids have, the stronger their G-quadruplex inductive ability.

benzo[1,3]dioxole group, telomeric DNA, G-quadruplex, alkaloids, Protoberberine, Benzophenanthridine

Citation: Yang S, Xiang J F, Yang Q F, et al. An important functional group, benzo[1,3]dioxole, of alkaloids induces the formation of the human telomeric DNA G-quadruplex. Chinese Sci Bull, 2011, 56: 613-617, doi: 10.1007/s11434-010-4257-2

The human telomere has attracted wide attention since it was found to exhibit a complicated relationship with tumor [1-4]. G-quadruplex, a type of secondary structure adopted by human telomeric DNA, has been shown to inhibit telomerase activity [5] which displays elevated levels of expression in $85 \%-90 \%$ of cancerous cells [4]. As a result, ligands with the ability to facilitate the formation or stability of G-quadruplexes have attracted great interest as a new class of potentially selective antitumor drugs [5-8].

Alkaloids are nitrogen-containing bases mostly produced by higher plants during metabolism. They occupy an important position in applied chemistry and also play an indispensable role in medicinal chemistry. A large number of alkaloids can form molecular complexes with nucleic acid structures, which might account for their biological activity [9].

The alkaloid groups Protoberberine and Benzophenanthridine have been found to exhibit antitumor activity.

*Corresponding authors (email: tangyl@iccas.ac.cn; jfxiang@iccas.ac.cn)
Some alkaloids from these two groups, such as Berberine, have been found to be G-quadruplex stabilizers and or can facilitate the formation of G-quadruplexes from singlestranded human telomeric DNA, such as (TTAGGG) (HT4) [10]. In this paper, the ability of five alkaloids from the two groups to induce G-quadruplexes was investigated. We found that the benzo[1,3]dioxole group in these alkaloids plays an important role in their G-quadruplex inductive abilities, and therefore detailed investigation of the benzo[1,3]dioxole group was carried out. Some factors influencing the benzo[1,3]dioxole group's role in inducing the formation of G-quadruplexes were also discussed.

Alkaloids Jat, Ber, Cop, San and Che (all at $98 \%$ purity 98\%) purchased from China Chengdu Scholar Bio-Tech. CO., LTD., and MA, MB, AMH, and EQC from Sigma, were used without further purification. All DNA oligonucleotides were obtained from Invitrogen (Beijing, China), purified by PAGE (purity 98\%) and used without further purification. Tris and EDTA (Beijing Chem. Co) were of analytical grade. 
Stock solutions of alkaloids were prepared by dissolving Jat, Ber, Cop, San, Che, MA, MB, AMH, and EQC in Tris- $\mathrm{HCl}$ buffer solution $\left(10 \mathrm{mmol} \mathrm{L}{ }^{-1}\right.$ Tris- $\mathrm{HCl}, 1 \mathrm{mmol} \mathrm{L}^{-1}$ EDTA, $\mathrm{pH}$ 7.4). Stock solutions of DNA oligonucleotides were prepared by dissolving DNA oligonucleotides in Tris- $\mathrm{HCl}$ buffer solution $\left(10 \mathrm{mmol} \mathrm{L}{ }^{-1}\right.$ Tris- $\mathrm{HCl}, 1 \mathrm{mmol} \mathrm{L}^{-1}$ EDTA, pH 7.4).

The CD data were collected on a Jasco-J 815 spectropolarimeter with a quartz cell of $1 \mathrm{~cm}$ optical path length. The final spectra were the averages of three repetitions. Each spectrum was baseline-corrected and the signal contributions of the Tris- $\mathrm{HCl}$ buffer solution were subtracted. The temperature of the cell holder was regulated by a JASCO PTC-423S temperature controller, and the cuvette-holding chamber was flushed with a constant stream of dry $\mathrm{N}_{2}$ gas to avoid water condensation on the cuvette exterior. Melting curves of the antiparallel G-quadruplexes were obtained by recording the $\mathrm{CD}$ intensity at $292 \mathrm{~nm}$. The heating rate was $1.0^{\circ} \mathrm{C} \mathrm{min}^{-1}$.

The samples were prepared by mixing a quantity of quaternary benzo[c]phenanthridine alkaloids (QBA) stock solution with a DNA oligonucleotide stock solution in Tris- $\mathrm{HCl}$ buffer solution $\left(10 \mathrm{mmol} \mathrm{L}{ }^{-1}\right.$ Tris- $\mathrm{HCl}, 1 \mathrm{mmol} \mathrm{L}{ }^{-1}$ EDTA, $\mathrm{pH}$ 7.4). The sample solutions were kept overnight at room temperature before measurement to the full reaction to occur.

CD measurement has been extensively applied to study secondary structures of DNA [11-13]. The CD spectral features allow the approximate, but convenient, recognition of different DNA motifs. Generally, HT4 alone presents an unfolded motif under salt-deficiency conditions, exhibiting a strong positive CD signal at $256 \mathrm{~nm}$ and a weak negative one at $280 \mathrm{~nm}$ in $10 \mathrm{mmol} \mathrm{L}^{-1}$ Tris- $\mathrm{HCl}$ buffer solution ( $\mathrm{pH}$ 7.4), which is the spectral feature of single-strand DNA (Figure 1, dashed line) [14].

To assess the effect of the functional group benzo[1,3] dioxole on the interactions of HT4, three alkaloids from the Protoberberine group: Jatrorrhizine (Jat) without, Berberine (Ber) with one, and Coptisine (Cop) with two benzo[1,3]dioxole groups (Chart 1), were tested their ability to induce HT4 folding into a G-quadruplex. The addition of Jat had little effect on the CD signal of HT4; while addition of Ber and Cop (Figure 1) led to the disappearance of the single-strand CD signals and appearance of two new peaks (a positive one at $292 \mathrm{~nm}$ and a negative one around 262 $\mathrm{nm}$ ), characteristic of an antiparallel G-quadruplex structure [14]. NMR results also supported the view that Ber and Cop could induce the formation of antiparallel G-quadruplexes (as shown in the Supporting information) Simply, Ber and Cop with a benzo[1,3]dioxole group could induce the formation of HT4 antiparallel G-quadruplexes, while Jat without this group could not. Moreover, the CD signals of G-quadruplexes induced by Cop (two benzo[1,3]dioxole groups) are stronger than those induced by Ber (only one group), indicating both the number and presence of a benzo[1,3]dioxole group might influence the inductive capacity of these alkaloids.
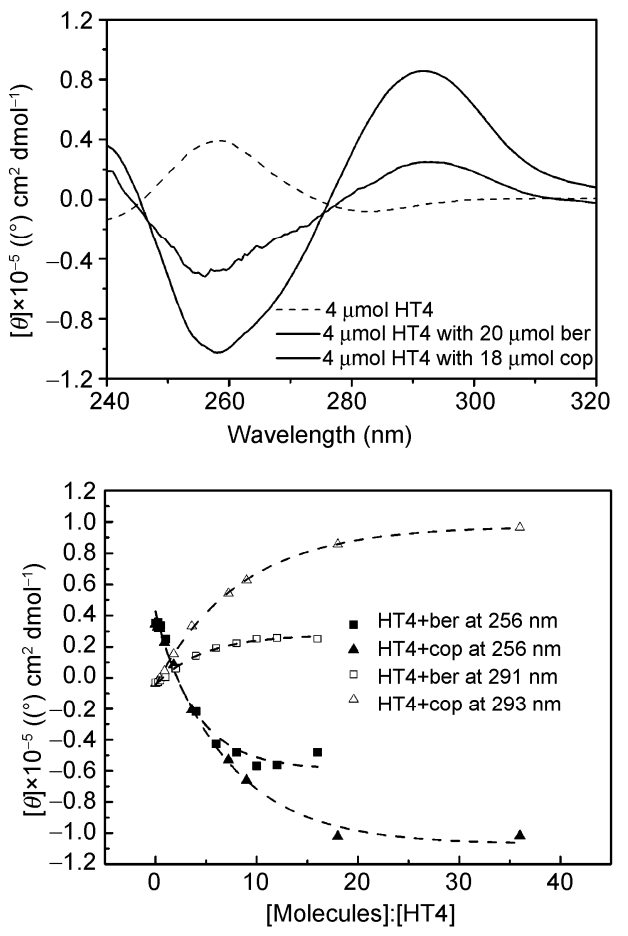

Figure 1 (a) The CD spectra of HT4 (dash line) and HT4 with Ber and Cop (solid lines). (b) The changes of the CD singles assigned to single-strand and antiparallel G-quadruplex HT4 against the ratio of [Molecule]: [HT4].

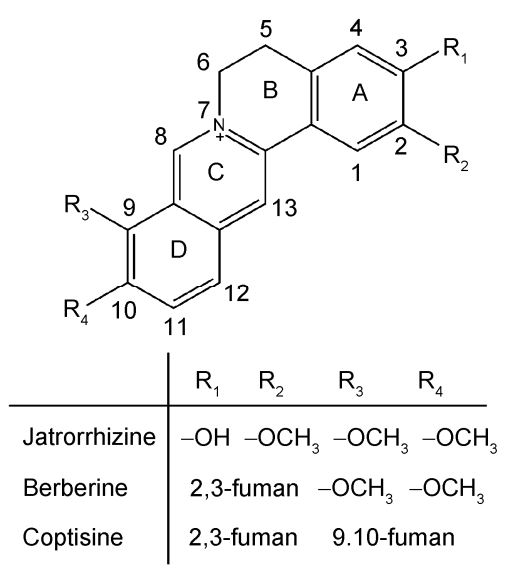

Chart 1 The molecular formula of Jat, Ber and Cop from Protoberberine group.

To further test this hypothesis, the interaction of HT4 with a pair of alkaloids from the Benzophenanthridine group, Chelerythrine (Che) with one and Sanguinarine (San) with two benzo[1,3]dioxole groups (Chart 2), was investigated. Addition of both Che and San (Figure 2) to single-stranded HT4 led to a decrease of the CD signals assigned to single-stranded DNA, and the appearance of positive CD signals around $290 \mathrm{~nm}$, attributed to antiparallel G-quadruplexes. This result implied that both Che and San could induce HT4 to fold into antiparallel G-quadru- 

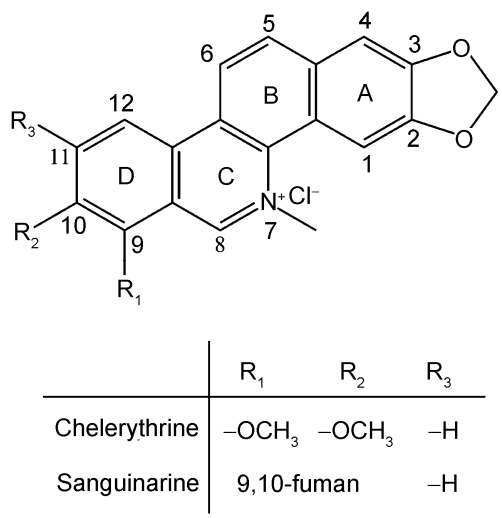

Chart 2 The molecular formula of Che and San from Benzophenanthridine group.
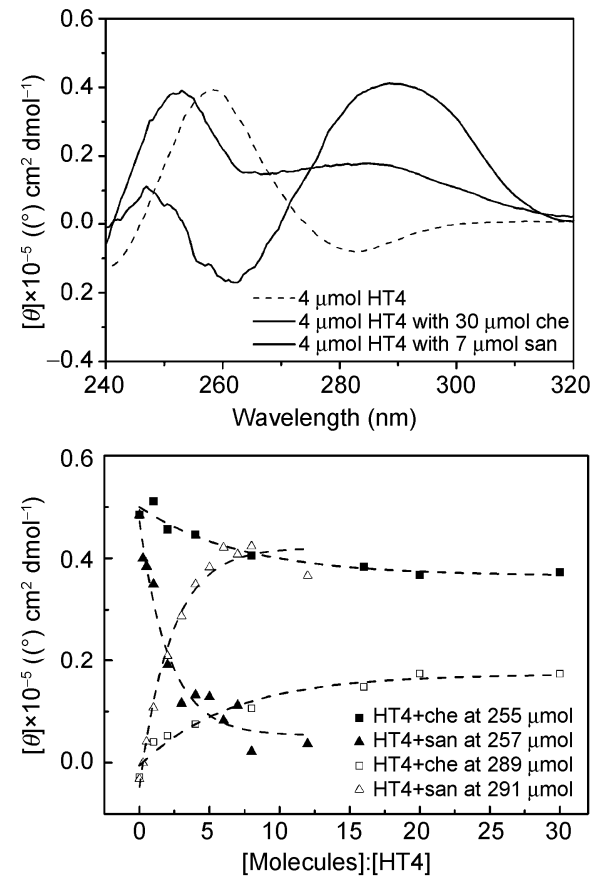

Figure 2 (a) The CD spectra of HT4 (dash line) and HT4 with Che and San (solid lines). (b) The changes of the CD signals assigned to single-strand and antiparallel G-quadruplex HT4 against the ratio of [molecule]: [HT4].

plexes. However, the spectral changes induced by Che were much weaker than those by San: the CD signals assigned to single-strand HT4 did not completely disappear and those assigned to G-quadruplex were weak, indicating Che has a weaker ability to induce changes in HT4. NMR results also showed that only San could induce the formation of antiparallel G-quadruplexes (as shown in Supporting information). The results are very similar as those of alkaloids from the Protoberberine group and demonstrate that benzo[1,3]dioxole is an important structural component of alkaloids that confers the capacity to induce the folding of human telomeric DNA into G-quadruplexes. The more functional groups the alkaloids have, the stronger their ability to induce G-quadruplexes.
The extended planar $\pi$-electron conjugated system is a necessary feature of most G-quadruplex ligands, especially the extended planar chromophore, which is similar in size and shape to that of a G-quartet stacked on its surface due to $\pi-\pi$ interactions. For example, telomestatin, a macrocyclic natural product comprising a similar structure to a G-tetrad, is currently regarded as a good G-quadruplex ligand and is the most efficient in vitro telomerase inhibitor [15-17]. Another important feature of G-quadruplex ligands is the central cationic aromatic core. It could promote their interaction with G-quadruplexes via interaction with the negative electrostatic center of the G-quadruplex by electrostatic interaction, thus attracting the G-quadruplex. It also acts as a "pseudo" potassium ion and is positioned above the centre of the G-quartets in the region of high negative-charge density. Primer studies have been reported, in which molecules, such as PIPER [18] and RHPS4 [19], with central cationic aromatic cores acting as "pseudo" potassium ions, were efficient G-quadruplex inducers and stabilizers. The alkaloids from the Protoberberine group and Benzophenanthridine group are also similar to the shape of a G-quartet and they have the central cationic aromatic cores, which suggested that their inductive ability might be attributable to $\pi-\pi$ stacking and a "pseudo" potassium ion, as discussed below.

Structurally, the presence of a benzo[1,3]dioxole group enlarges the planar $\pi$-electron conjugated system and strengthens the $\pi-\pi$ interactions between a G-quartet and its ligands. Furthermore, the overlap between the $\pi$ system of these alkaloids and the complementary G-quartet of the G-quadruplex suggests that the A-ring benzo[1,3]dioxole group causes the alkaloids of the Protoberberine group and Benzophenanthridine group to have a more similar shape to that of a G-quartet, which might make them to be more efficient G-quadruplex ligands. Further investigation was carried out involving more molecules: 3',4'-(methylenedioxy)aniline (MA), 3', $4^{\prime}$-(methylenedioxy)benzylamine (MB), 2'-amino-4',5'-methylenedioxyacetophenone hydrochloride (AMH), and ethyl 8-chloro[1,3]dioxolo[4,5-g]quinoline-7carboxylate (EQC) (Chart 3).

They all have the benzo[1,3]dioxole group with positively charged centers $\mathrm{N}^{+}$nearby, a common feature of G-quadruplex ligands. An effect of the $\mathrm{N}^{+}$position should be gained from MB and MA, and an effect of the substitutions around benzo[1,3]dioxole should be gained from AMH with the acetyl group, and EQC with the pyridine ring enlarging the $\pi$-conjugated system. Unfortunately, the experimental results showed that none of them could induce HT4 to fold into a G-quadruplex. Thus, for small molecules with $\pi$-aromatic surfaces of less than two rings, neither the position of the positively charged centers $\mathrm{N}^{+}$nor the substitutions could adjust their ability to facilitate the formation of G-quadruplexes. These results suggest that benzo[1,3] dioxole cannot play a role in the process of the G-quadruplex induction by itself, and it must act as a part of a large electron deficient $\pi$-aromatic surface, which together might 
<smiles>[R]c1cc2c(cc1[R])OCO2</smiles>

\begin{tabular}{|c|c|c|}
\hline & R1 & R2 \\
\hline MA & $-H$ & $-\mathrm{NH}_{2}$ \\
\hline MB & $-\mathrm{H}$ & $-\mathrm{CH}_{2} \mathrm{NH}_{3}$ \\
\hline AMH & $\begin{array}{c}\mathrm{O} \\
-\mathrm{C}-\end{array}$ & $-\mathrm{NH}_{2} \mathrm{HCl}$ \\
\hline EQC & & \\
\hline
\end{tabular}

Chart 3 The molecular formula of MA, MB, AMH and EQC.

enhance the inductive ability of such compounds.

The positive CD signal at $\sim 256 \mathrm{~nm}$ is assigned to single-stranded HT4. Thus, the comparison of the change of the CD signal of this single-stranded HT4 before and after the addition of ligands reflects the change in the amount of single-stranded HT4 in specific system. As shown in Figures 1 and 2, the changed signal at $256 \mathrm{~nm}$ showed that the addition of a small amount of Cop and Ber, for example, at less than 4 times of the amount of HT4, could lead to the complete disappearance of the single-strand HT4 signal. This indicates that both Cop and Ber have strong abilities to induce the formation of G-quadruplexes, with Cop having a stronger ability than Ber. Similarly, the addition of San caused the almost complete disappearance of the singlestranded HT4 signal transform it the signal for antiparallel G-quadruplexes. In contrast, the addition of Che only induced the disappearance of a small amount of single-strand HT4 signal, indicating that San has a stronger ability to induce G-quadruplexes than Che. Meanwhile, we applied circular dichroism to monitor the melting temperature $\left(T_{\mathrm{m}}\right)$ of induced G-quadruplexes at $292 \mathrm{~nm}$ (Figures 3 and 4). The change in $T_{\mathrm{m}}$ in the folded and unfolded quadruplex structures upon interacting with the ligands provides evidence of thermal stabilization of the DNA structure. As shown in Figures 3 and 4, the $T_{\mathrm{m}}$ of G-quadruplexes induced by Cop, Ber, San, and Che are about 54, 44, 42 and $32^{\circ} \mathrm{C}$, respectively, indicating that the G-quadruplexes induced by Cop were more stable than those induced by Ber, and the G-quadruplexes induced by San were more stable than those induced by Che. Both of the above experiments led to the conclusion that the number of benzo[1,3]dioxole groups could influence the inductive ability of these series of alkaloids. Furthermore, we found that Che had less inductive ability than Ber, despite having the same single benzo[1,3]dioxole group. San, with two benzo[1,3]dioxole groups had an equivalent inductive ability to Ber. To the best of our knowledge, most of the G-quadruplex ligands
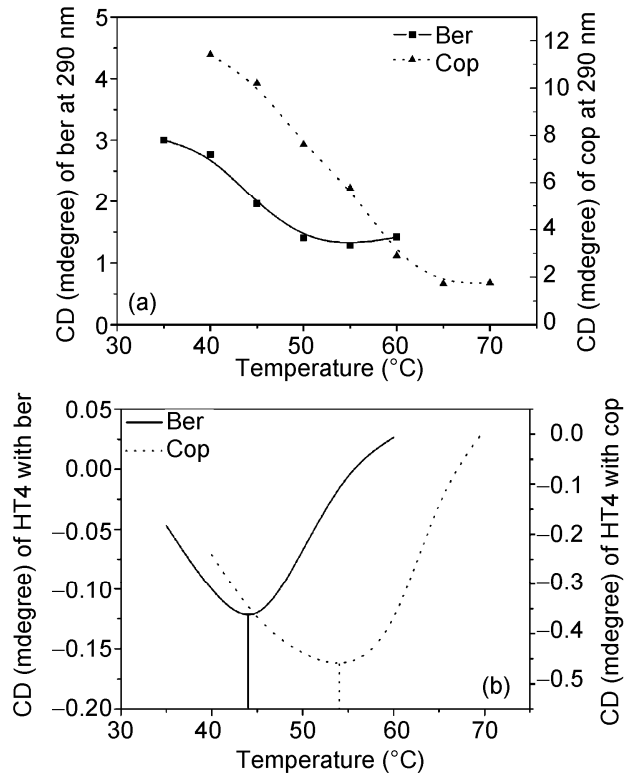

Figure 3 (a) CD melting profiles of $4 \mu \mathrm{mol} / \mathrm{L}$ HT4 G-quadruplex and 4 $\mu \mathrm{mol} / \mathrm{L}$ HT4 with $20 \mu \mathrm{mol} / \mathrm{L}$ Cop and Ber at $292 \mathrm{~nm}$. (b) The second derivative curves of the changes of CD signals of $4 \mu \mathrm{mol} / \mathrm{L}$ HT4 G-quadruplex and $4 \mu \mathrm{mol} / \mathrm{L}$ HT4 with $20 \mu \mathrm{mol} / \mathrm{L}$ Cop and Ber at $292 \mathrm{~nm}$ against the temperature.
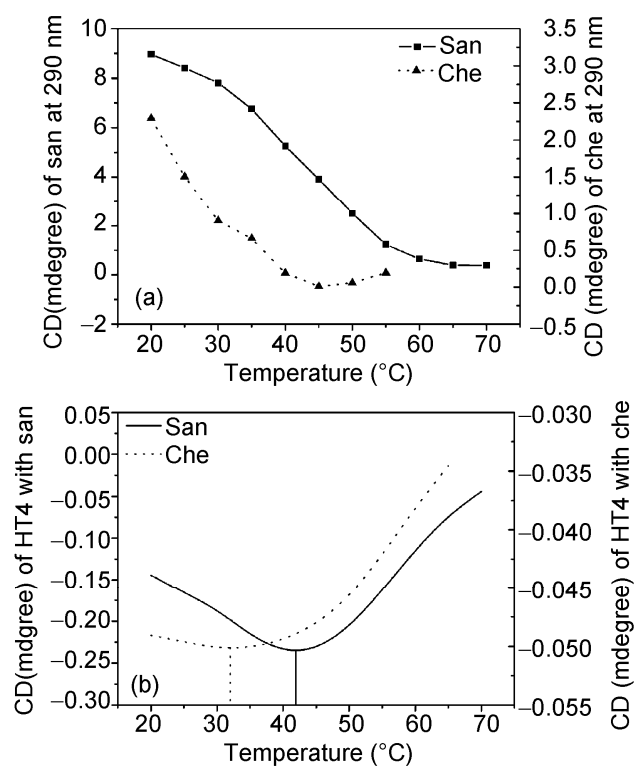

Figure 4 (a) CD melting profiles of $2 \mu \mathrm{mol} / \mathrm{L}$ HT4 G-quadruplex and 2 $\mu \mathrm{mol} / \mathrm{L}$ HT4 with $20 \mu \mathrm{mol} / \mathrm{L}$ San and Che at $292 \mathrm{~nm}$. (b) The second derivative curves of the changes of CD signals of $2 \mu \mathrm{mol} / \mathrm{L}$ HT4 G-quadruplex and $2 \mu \mathrm{mol} / \mathrm{L}$ HT4 with $20 \mu \mathrm{mol} / \mathrm{L}$ San and Che at $292 \mathrm{~nm}$ against the temperature.

have extended planar $\pi$-electron conjugated systems conducive to the formation of G-quartets. For the alkaloids tested here, the extended planar chromophores of the Protoberberine group alkaloids are not conjugated as well as those of the Benzophenanthridines, and they should, therefore, have less inductive ability; however, in our experiments, they 
demonstrated better abilities. From their structures, we could see that San and Che have a methyl at the $7-\mathrm{N}^{+}$, while Cop and Ber do not. We hypothesize that when the random HT4 were attacked by the central cationic aromatic core, they could approach from all directions of Cop; from all directions except the D-ring direction of Ber; from only two sides, avoiding the methoxyl groups A-ring and D-ring, of Jat; from all directions except the methyl direction at the $7-\mathrm{N}^{+}$of San; and from only the A-ring direction avoiding the methoxyl groups at the D-ring and the methyl group at $7-\mathrm{N}^{+}$of Che. Thus the lower number of directions the random HT4 can be approached by, the lower ratio the G-quadruplex is formed. These experimental results indicate that Che probably forms a steric hindrance around their A-ring benzo[1,3]dioxole, while Cop and Ber do not. Although San also has this methyl group, there is still a D-ring benzo[1,3]dioxole to prevent its ability to form G-quadruplexes from being so influenced. This is another explanation why San, with two benzo[1,3]dioxole groups, has the equivalent inductive ability as Ber. This leads to the conclusion that steric hindrance near the benzo[1,3]dioxole group plays a negative role in the G-quadruplex induction.

Benzo[1,3]dioxole is an important structural part of alkaloids that induce human telomeric DNA to fold into G-quadruplex. Jat, with no benzo[1,3]dioxole group in its structure cannot induce single-strand HT4 to fold into G-quadruplexes. Ber with one benzo[1,3]dioxole group showed an inductive ability that was not as strong as Cop, which has two benzo[1,3]dioxole groups. Similar results were found for the Benzophenanthridine group, in which Che, with only one benzo[1,3]dioxole group in its structure, had not as strong an inductive ability as San. Thus the more functional groups in an alkaloid, the stronger its ability to induce the formation of G-quadruplexes. In addition, benzo$[1,3]$ dioxole cannot induce G-quadruplex formation by itself, it must act as a part of a large electron deficient $\pi$-aromatic surface, which can enhance the inductive ability of such compounds. Moreover, steric hindrance, such as the methyl at the $7-\mathrm{N}^{+}$, around benzo[1,3]dioxole, is probably responsible for the reduced inductive ability of Che compared with Ber and San. Thus, careful examination of the structural effects of G-quadruplex ligands on their properties is necessary to further the search and design of efficient antitumor agents.

1 De Cian A, Lacroix L, Douarre C, et al. Biochimie, 2008, 90: 131-155

Blackburn E H. Cell, 2001, 106: 661-673

3 Riou J F, Guittat L, Mailliet P, et al. Proc Natl Acad Sci USA, 2002, 99: 2672-2677

4 Kim N W, Piatyszek M A, Prowse K R, et al. Science, 1994, 266 : 2011-2015

5 Davis J T. Angew Chem Int Ed Engl, 2004, 43: 668-698

6 Ou T M, Lu Y J, Tan J H, et al. Chem Med Chem, 2008, 3: 690-713

7 Monchaud D, Teulade-Fichou M P. Org Biomol Chem, 2008, 6: 627-636

8 Zhou Q, Li L, Xiang J, et al. Angew Chem Int Ed Engl, 2008, 47: 5590-5592

9 Maiti M, Kumar G S. Med Res Rev, 2007, 27: 649-695

10 Bai L P, Hagihara M, Jiang Z H , et al. Chem Bio Chem, 2008, 9: 2583-2587

11 Li J, Correia J J, Wang L, et al. Nucleic Acids Res, 2005, 33: 4649-4659

12 Rujan I N, Meleney J C, Bolton P H. Nucleic Acids Res, 2005, 33 : 2022-2031

13 Ambrus A, Chen D, Dai J, et al. Nucleic Acids Res, 2006, 34: 2723-2735

14 Balagurumoorthy P, Brahmachari S K. J Biol Chem, 1994, 269: 21858-21869

15 Shin-Ya K, Wierzba K, Matsuo K, et al. J Am Chem Soc, 2001, 123 : 1262-1263

16 Kim M Y, Vankayalapati H, Shin-Ya K, et al. J Am Chem Soc, 2002, 124: 2098-2099

17 Seenisamy J, Bashyam S, Gokhale V, et al. J Am Chem Soc, 2005, 127: 2944-2959

18 Fedoroff O Y, Salazar M, Han H, et al. Biochemistry, 1998, 37: 12367-12374

19 Gavathiotis E, Heald R A, Stevens M F, et al. Angew Chem Int Ed Engl, 2001, 40: 4749-4751

Open Access This article is distributed under the terms of the Creative Commons Attribution License which permits any use, distribution, and reproduction in any medium, provided the original author(s) and source are credited.

\section{Supporting Information}

Figure S1 The CD spectra of HT4 with Jat.

Figure S2 The CD spectra of HT4 with MA.

Figure S3 The CD spectra of HT4 with MB.

Figure S4 The CD spectra of HT4 with AMH.

Figure S5 The CD spectra of HT4 with EQC.

Figure S6 NMR spectra of telomeric ssDNA and that with Cop, Ber, San or in Na+ solution.

The supporting information is available online at csb.scichina.com and www.springerlink.com. The supporting materials are published as submitted, without typesetting or editing. The responsibility for scientific accuracy and content remains entirely with the authors. 